\title{
COVID-19 dominates discussions at AAN 2021
}

Following its cancellation owing to the COVID-19 pandemic in 2020, the American Academy of Neurology (AAN) Annual Meeting made a welcome return in a virtual format in April 2021. Not surprisingly, the impact of the pandemic on the neurology community was a recurring theme. The Hot Topics Plenary Session was devoted entirely to COVID-19 and included contributions from prominent medical experts such as Anthony Fauci and Walter Koroshetz.

The scientific programme featured numerous presentations on the neurological complications of COVID-19. For example, Sherry Chou introduced the work of the GCS-NeuroCOVID Consortium, a worldwide collaboration that is exploring the prevalence, mechanisms and outcomes of COVID-19-related neurological symptoms. Omar Abdel-Mannan described an immune-mediated disorder termed PIMS-TS, which is observed in children after SARS-CoV-2 infection and manifests with neurological symptoms in around $50 \%$ of cases.
The effects of the COVID-19 pandemic on neurological care - in particular, stroke care - were also examined at AAN 2021. Thanh Nguyen reported on a large international study that demonstrated a global decline in hospitalization and intravenous thrombolysis for stroke during the pandemic. In addition, Nicte Mejia discussed how the pandemic has exposed and amplified racial disparities in stroke care.

Finally, speaking in the Hot Topics Plenary Session, Serena Spudich provided a glimpse of future directions in COVID-19 research, highlighting ongoing studies on neurological syndromes in the post-acute phase of the disease. This emerging issue will undoubtedly occupy neurologists for years to come as the long-term consequences of the pandemic become increasingly apparent.

Heather Wood

RELATED ARTICLES Pezzini, A. \& Padovani, A. Lifting the mask on neurological manifestations of COVID-19. Nat. Rev. Neurol. 16, 636-644 (2020) | Nolen, L. \& Mejia, N. I. Inequities in neurology amplified by the COVID-19 pandemic. Nat. Rev. Neurol. 17, 67-68 (2021)

\section{Racial differences in the response to multiple sclerosis therapy}

Preliminary data presented at the American Academy of Neurology (AAN) Virtual Annual Meeting (17-22 April 2021) indicate that the response to anti-CD20 B cell-depleting therapies differs between Black and white individuals. Anti-CD20 monoclonal antibodies, including rituximab and ocrelizumab, are commonly used to treat autoimmune neurological diseases such as multiple sclerosis (MS) and neuromyelitis optica spectrum disorder (NMOSD).

The aim of the research, which was conducted by llya Kister and colleagues at the NYU Grossman School of Medicine, New York, NY, USA, was to measure blood $B$ cell repopulation following rituximab or ocrelizumab infusion in people with MS or NMOSD. The study cohort included 61 individuals who identified as Black and 60 individuals who identified as white.

At 4-6 months after treatment, the proportion of individuals showing evidence of $B$ cell repopulation was similar between the Black and white participants ( $20.8 \%$ versus $17.9 \%$ ).
Between 6 and 12 months, however, the proportion was considerably higher in the Black patients (76.2\%, compared with $33.3 \%$ in white patients), suggesting a more rapid rate of $B$ cell repopulation in this group.

"Our findings raise the question of whether the same therapy dose may be equally effective for all people, and that could have implications for the way Black people with autoimmune diseases like MS and NMOSD are treated in the future," Kister concluded.

The investigators acknowledge that the study was limited by inconsistencies in the timings of the B cell measurements after drug infusion. Nevertheless, the results suggest that further research is warranted to examine whether $B$ cell repopulation rates following anti-CD20 therapy reflect racial differences in disease activity.

Heather Wood

RELATED ARTICLE Tintore, M. et al. Treatment of multiple sclerosis - success from bench to bedside. Nat. Rev. Neurol. 15, 53-58 (2019)

\section{AAN 2021}

\section{A promising new therapy for ALS}

The investigational drug AMX0035 has beneficial effects on survival and function in people with amyotrophic lateral sclerosis (ALS), according to data presented at the American Academy of Neurology (AAN) Virtual Annual Meeting (17-22 April 2021). Sabrina Paganoni reported on the latest findings from the CENTAUR trial, building on data already published in 2020.

AMX0035 is a coformulation consisting of sodium phenylbutyrate, which targets endoplasmic reticulum stress, and taurursodiol, which targets mitochondrial dysfunction. The two drugs individually exhibit neuroprotective properties in experimental models of ALS and are expected to have additive or even synergistic effects when used in combination.

The CENTAUR trial recruited 137 patients $\leq 18$ months after ALS onset. Participants were randomly assigned in a 2:1 ratio to receive AMX0035 or placebo for 24 weeks. This initial period was followed by an open-label extension (OLE) in which all participants received AMX0035. As Paganoni explained, the randomized period was intentionally kept short to maximize the number of patients who survived long enough to enter the OLE.

After 24 weeks, the AMX0035 group showed better function on the ALS Functional Rating Scale - Revised than the placebo group. However, this period was too short to detect significant differences in survival.

Over 3 years, the risk of death was $44 \%$ lower in the original AMX0035 group than in the original placebo group. The former group survived for a median of 11.5 months longer than predicted, compared with 6.4 months in the latter group. In addition, survival times before tracheostomy, permanent assisted ventilation and first hospitalization were longer in the original AMX0035 group than in the original placebo group.

The data indicate that the CENTAUR trial provides meaningful survival data despite the brevity of the randomized period, and that AMX0035 can be beneficial even when administered after a 6-month delay.

Heather Wood

RELATED ARTICLES Paganoni, S. et al. Trial of sodium phenylbutyrate-taurursodiol for amyotrophic lateral sclerosis. N. Engl. J. Med. 383, 919-930 (2020)| Paganoni, S. et al. Longterm survival of participants in the CENTAUR trial of sodium phenylbutyrate-taurursodiol in amyotrophic lateral sclerosis. Muscle Nerve 63, 31-39 (2021) 